\title{
Angioinvasive Pulmonary Aspergillosis After Allogeneic Bone Marrow Transplantation: Clinical and High-Resolution Computed Tomography Findings in 12 Cases.
}

Emerson L. Gasparetto ${ }^{1}$, Carolina A. Souza ${ }^{1}$, Priscilla Tazoniero', Taisa Davaus ${ }^{1}$, Dante L. Escuissato ${ }^{1}{\text { and } \text { Edson }^{1} \text { Marchiori }}^{2}$ ${ }^{1}$ University of Paraná, Curitiba, PR; ${ }^{2}$ University of Rio de Janeiro, Rio de Janeiro, RJ, Brazil

\begin{abstract}
The aim of this study was to present the clinical and high-resolution CT scan findings of angioinvasive pulmonary aspergillosis (APA) in 12 patients who underwent allogeneic bone marrow transplantation (BMT). The CT scans were reviewed by three chest radiologists who assessed the pattern and distribution of findings by consent. There were $7(58 \%)$ female and $5(42 \%)$ male patients, with aging between 5 and 50 years (average of 26 years). All patients were submitted to BMT for the treatment of hematological conditions. The diagnosis of APA was defined between 5 and 373 days after BMT, with average of 111 days. Three cases $(25 \%)$ were diagnosed in the neutropenic phase after the BMT, five $(42 \%)$ in the early phase and four patients in the late phase post-BMT. Regarding high-resolution CT (HRCT) scan findings, nodules were found in $75 \%$ of the cases (9/12), most of the cases with more than 10 lesions (7/ 9) and of centrilobular localization (6/9). Consolidations were identified in seven patients (58\%), being single in six, and commonly presenting ill defined borders $(n=3)$ and subsegmental localization $(n=5)$. Ground glass attenuation was found in six patients (50\%). The halo sign was observed in nine cases $(\mathbf{7 5 \%})$. Cavitations were seen in two airspace consolidations and one large nodule $(2.5 \mathrm{~cm})$. Patients submitted to BMT presenting respiratory symptoms and nodules or consolidations with halo sign at HRCT scan need to have the diagnosis of angioinvasive pulmonary aspergillosis included in all the post BMT phases.

Key-Words: Invasive pulmonary aspergillosis, bone marrow transplantation, high-resolution computed tomography.
\end{abstract}

Bone marrow transplantation (BMT) is a potentially curative alternative of treatment for diseases such as acute leukemia, chronic mielogenous leukemia, aplastic anemia and Fanconi's anemia. Despite increasing survival rates related to this procedure, pulmonary complications are still common and constitute an important cause of morbidity and mortality. Respiratory complications occur in $40 \%$ to $60 \%$ of patients after BMT and are major causes of morbidity and mortality. These complications reflect the immunologic status of the patients and occur in three phases: 1) neutropenic phase: immediately after transplantation, characterized by a period of profound neutropenia lasting 2-3 weeks; 2) early phase: period until 100 days after BMT with a gradual recovery of neutrophils and a lessening of immune impairment; 3) late phase: 100 days after BMT when the immune status has been recovered [1,2].

Fungal pneumonia is a common cause of respiratory complications post-BMT, generally occurring in the neutropenic phase [3,4]. Nowadays, the Aspergillus sp. is the commonest agent of fungal pneumonia post-BMT, being responsible for until $90 \%$ of these infections [5]. In these patients, aspergillus infection occurs in its angioinvasive pulmonary form (APA), and the diagnosis can be settled through sputum cultures, bronchoalveolar lavage or blood cultures, as well as through tissue examination of lung fragments obtained by biopsy [6-10]. Laboratorial confirmation of the diagnosis can delay the therapy onset, what can be fatal to these patients since this infection tends

Received on 7 July 2006; revised 17 November 2006.

Address for correspondence: Dr. Emerson L. Gasparetto. Serviço de Radiologia Médica - HC - UFPR. Rua General Carneiro 181. Zip code: 80060-900 - Curitiba, PR. E-mail: gasparetto@hotmail.com.

The Brazilian Journal of Infectious Diseases 2007;11(1):110-113. (C) 2007 by The Brazilian Journal of Infectious Diseases and Contexto Publishing. All rights reserved. to present fulminating evolution. Therefore, the importance of imaging investigation in such patients is widely emphasized in the literature, mostly through computed tomography (CT) scan, with the goal of identifying aspects that could suggest this diagnosis and authorizing early onset of anti-fungal therapy. The most common high resolution CT (HRCT) scan findings in patients with APA include nodules with surrounding ground glass attenuation ("halo sign”), consolidations and "air-crescent sign" [9,11,12].

The authors report 12 cases of APA post-BMT with emphasis to the clinical and HRCT scan findings.

\section{Materials and Methods}

The medical charts, files and HRCT scans of patients submitted to allogeneic BMT in the Hospital de Clinicas of the University of Paraná between 1990 and 2003 were reviewed, and 29 cases with clinical and radiological suspicion of fungal pneumonia were found. Among them, 12 had confirmed diagnosis of APA and were included in the study. The presence of Aspergillus sp. in sputum culture (2/12), bronchoalveolar lavage culture (LBA) (3/12), lung biopsy (2/12) or microscopic findings of fungus infection in necropsy (5/12) were considered diagnosis of APA. Cases of APA associated with other pulmonary infections and those with clinical and radiological suspicion of IPA, but without laboratorial confirmation, were excluded of the study.

The time between the HRCT scan and the diagnosis of APA varied from 1 to 7 days. All the HRCT were performed at end-inspiration using $2 \mathrm{~mm}$ collimation at $10 \mathrm{~mm}$ intervals (Somaton ART, Siemens, Germany). Images were photographed at mediastinal (width, $400 \mathrm{H}$; level, $20 \mathrm{H}$ ) and lung (width, 1500 $\mathrm{H}$; level, $-700 \mathrm{H}$ ) window settings. The films were studied by three radiologists in different times, and final decision was obtained by consensus. The following HRCT scan findings 
were assessed: distribution of the lesions (central and/or peripheral, unilateral or bilateral, symmetrical or asymmetrical, and upper/middle/lower zones distribution), pattern of abnormality (ground-glass and air-space opacities, bronchial dilatation, bronchial wall thickening, tree-in-bud opacities, reticular opacities, large or small nodules, mosaic perfusion and vessel attenuation), lymph node enlargement, pleural effusions, as well as any other lung abnormalities.

Nodules were classified according to their dimensions as smaller than $1 \mathrm{~cm}$, between 1 and $3 \mathrm{~cm}$ and bigger than $3 \mathrm{~cm}$, and according to the number in less than 5, between 5 and 10 or more than 10 , being then classified regarding their localization as centrilobular, peribronchovascular or random. Consolidations were classified as single site or multifocal, well or ill defined borders and from subsegmental, peribronchovascular or random localization. Multifocal areas of ground glass attenuation were reviewed according to their centrilobular, peribronchovascular or random localization. Presence of the halo sign and cavitations, with or without air- crescent sign were also studied.

Clinical data were obtained through medical charts review and included the time between the BMT and the diagnosis of APA, signs and symptoms, neutrophil and platelet count, presence of graft versus host disease (GVHD), acute or chronic, use of corticosteroids and prophylaxis with fluconazole.

Clinical follow-up of all patients was included until death or until the moment of the study conclusion. Death related to APA were considered when occurred until one month after the HRCT scan and related to respiratory failure.

\section{Results}

There were 7 (58\%) female and 5 (42\%) male patients, with aging between 5 and 50 years (average of 26 years). All the patients were submitted to BMT for the treatment of hematological conditions. Six of them had severe aplastic anemia, four chronic myelogenous leukemia, one acute myeloid leukemia and one had acute lymphocytic leukemia.

The diagnosis of APA was defined between 5 and 373 days after BMT, with average of 111 days. Three cases (25\%) were diagnosed in the neutropenic phase after the BMT, five (42\%) in the early phase and four patients (33\%) had the diagnosis in the late phase post-BMT.

The mean signs and symptoms were fever (100\%), dry or productive cough (42\%), dyspnea (25\%) and chest pain (17\%). Nine patients presented chronic or acute GVHD affecting skin, liver or bowels, all of them with histological confirmation. In most of the cases (83\%) the APA occurred during corticosteroids administration and eight patients were using fluconazole as prophylaxis. Neutrophil count in the moment of the HRCT examination was between 50 and $8,500 / \mathrm{mm}^{3}$, with average of $2,348 / \mathrm{mm}^{3}$. Platelet count ranged between 15,000 and $162,000 / \mathrm{mm}^{3}$.

Regarding HRCT scan findings, nodules were found in $75 \%$ of the cases (9/12), most of the cases with more than 10 lesions (7/9) and of centrilobular localization (6/9) (Figures 1A and 1B). In all cases there were nodules which were smaller than $1 \mathrm{~cm}$, and in six cases nodules with dimensions between 1 and $3 \mathrm{~cm}$ were seen. Nodules whose dimensions were bigger than $3 \mathrm{~cm}$ were seen in 3 cases, all of them single and with peribronchovascular localization. Cavitation and tree-in-bud pattern were found in one case each one (Figures 2A and 2B).

Consolidations were identified in seven patients (58\%), being single in six, and commonly presenting ill defined borders $(\mathrm{n}=3)$ and subsegmental localization $(\mathrm{n}=5)$ (Figures $3 \mathrm{~A}$ and 3B). Ground glass attenuation was found in six patients (50\%), being of peribronchovascular localization in four, random in one and centrilobular in another case. The halo sign was observed in nine cases (75\%), four of then surrounding consolidations and five surrounding nodules. Cavitations were seen in two air-space consolidations and one nodule $(2.5 \mathrm{~cm})$. In three cases bronchiectasis /bronchiolectasis were seen associated with areas of mosaic perfusion, denoting the presence of small airway disease. All these patients had clinical and pulmonary function tests corroborating this diagnosis.

The majority of patients (75\%) presented more than one pattern of abnormality at the HRCT scan. The most common association was areas of consolidation and nodules, being found in four of the twelve cases. In three patients nodules were seen associated to areas of ground glass attenuation.

Bilateral and asymmetric distribution of lesions was the dominant pattern (8/12). In two cases lesions were symmetric and bilateral, and in two patients the lesions were confined to one of the lungs. Distribution between superior, middle or inferior thirds was similar and there was no predominance for any of these regions.

Nine patients died due to the APA, with mean survival time of 19 days. The other three patients achieved resolution of the pulmonary infection. Two of then died 53 and 245 days after HRCT, without evidence of persistency of IPA, and one remained well until conclusion of the study.

\section{Discussion}

The three different periods after BMT can be divided in three different phases. The first, also known as neutropaenic, is characterized by pulmonary infections, alveolar hemorrhage, pulmonary edema and drug reactions. At the second phase, or early phase, infections by cytomegalovirus (CMV) and respiratory syncytial virus are common. The third phase, or late phase, is characterized by the occurrence of bronchiolitis obliterans, cryptogenic organizing pneumonia and chronic GVHD [3-5]. Invasive pulmonary aspergillosis occurs more often after the neutropenic phase [5]. In this study, pulmonary infection by Aspergillus sp. was diagnosed in the neutropenic phase in $25 \%$ of the cases, in the early phase in $42 \%$ and in the late phase in $33 \%$ of the patients.

Aspergillus sp. is the main cause of fungal pneumonia after BMT and its incidence ranges between 4 and 13\%. Risk factors described for the development of APA include more than three weeks of corticosteroids therapy, neutropenia and GVHD [4,13]. In the present study, 83\% of the patients were in prolonged usage of corticosteroids in the moment of IPA 
Figures 1A and B. 32 year-old male patient with IPA 42 days after BMT. High-resolution CT (pulmonary (A) and mediastinal (B) windows) showing an air-space consolidation with surrounding ground-glass attenuation (halo sign), in the right superior lobe.
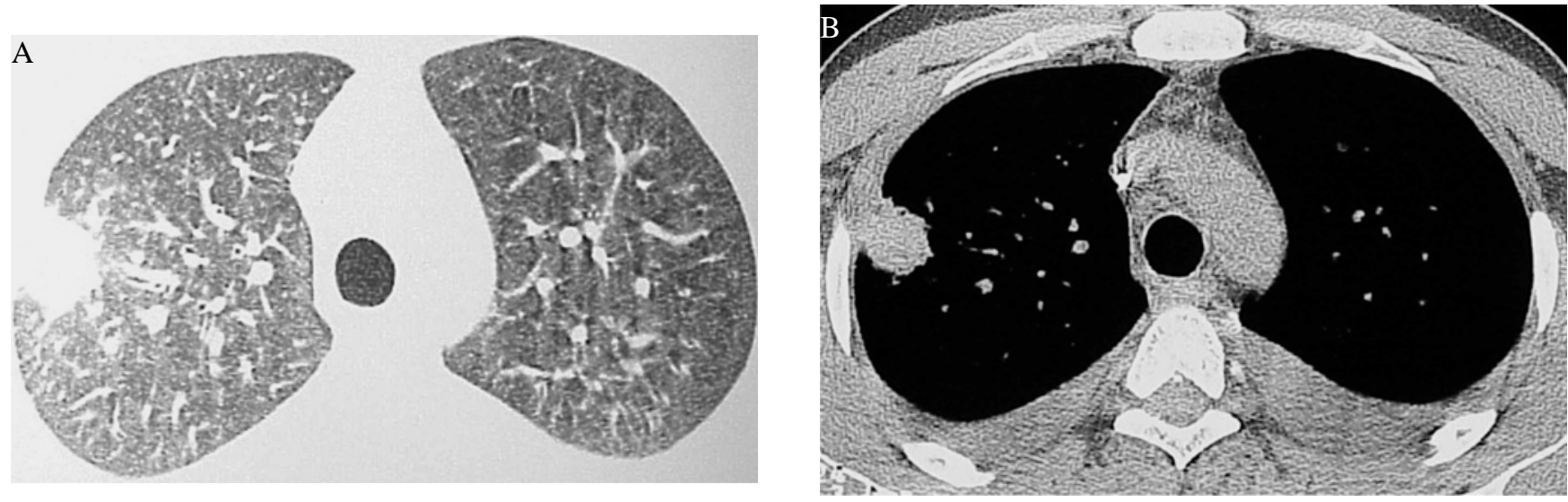

Figures 2A and B. 12 year-old female patient with IPA 19 days after BMT. High-resolution CT demonstrating in A an irregular opacity in the right inferior lobe, associated to bilateral small centrilobular nodules and foci of ground-glass attenuation. In B, an escavated nodule with septations in the middle lobe, also associated to bilateral small centrilobular nodules and foci of groundglass attenuation.
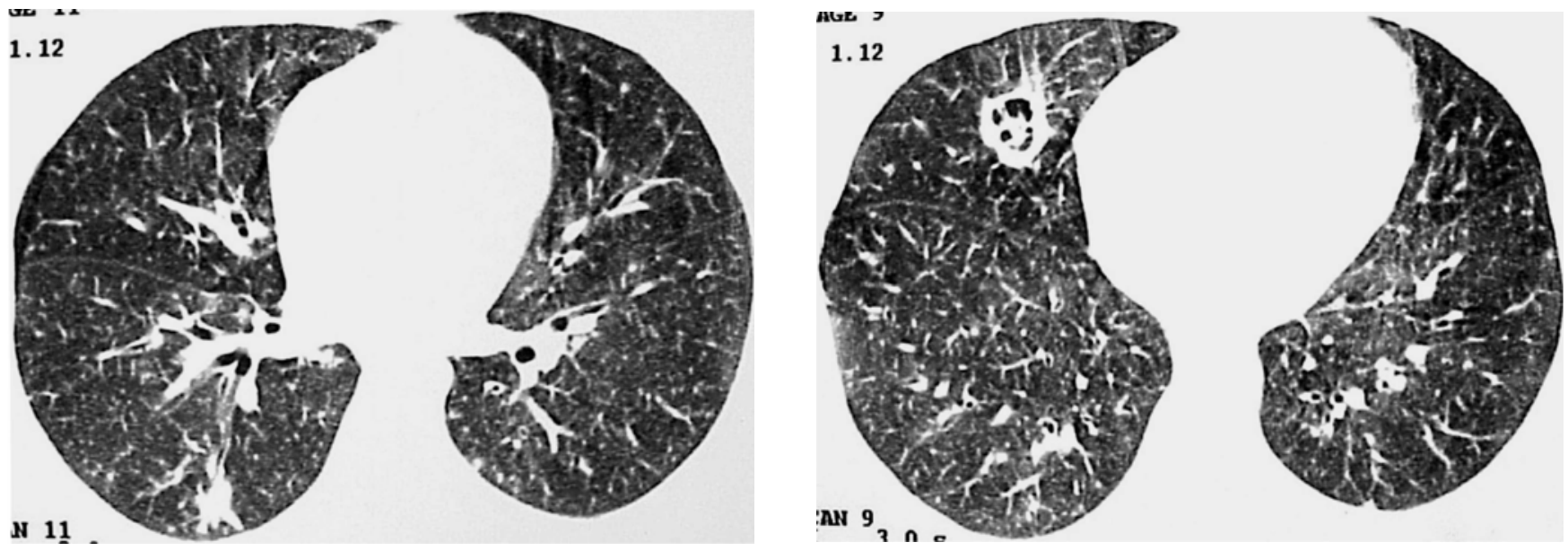

Figures 3A and B. 45 year-old male patient with IPA 62 days after BMT. High-resolution CT (pulmonary (A) and mediastinal (B) windows) demonstrating an air-space consolidation with surrounding ground-glass attenuation (halo sign), in the right inferior lobe.
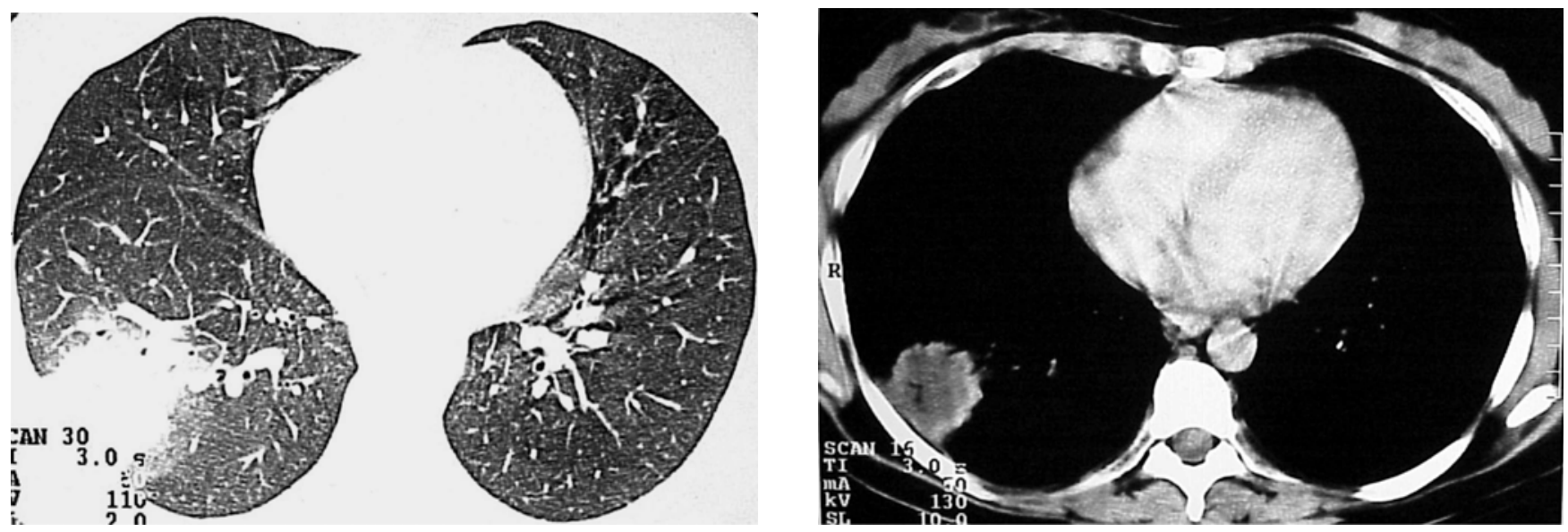
diagnosis, 75\% had a confirmed diagnosis of GVHD and 33\% were neutropenic.

The HRCT scan is superior to plain radiographic films in the detection and characterization of pulmonary complications after BMT. This exam is indicated in the presence of clinical manifestations of pulmonary disease with normal or unspecific radiographic findings, and for those patients who do not present clinical improvement despite adequate therapy [9,14-17]. Therefore, the HRCT scan allows more effective investigation for patients in the period after BMT, which is of high importance in such patients whose clinical deterioration is fast and lethal.

The HRCT scan findings of APA include nodules with surrounding ground glass area (the halo sign) and areas of sub-pleural consolidation, which corresponds to pulmonary infarction. In severe neutropenic patients the halo sign is highly suggestive of APA. However, other diseases associated or not to the BMT, such as candida infection, CMV pneumonia, herpes simplex infection, Wegener's granulomatosis, Kaposi's sarcoma and hemorrhagic metastasis can also present with this finding [16,17]. If there is a separation between necrotic lung fragments and the adjacent parenchyma, the air crescent sign can be seen. This sign is observed in patients with APA during the convalescent phase (2 to 3 weeks after therapy onset and neutropenia resolution) [18].

In the present study, the most common HRCT scan findings were small centrilobular nodules (75\%), air-space consolidations (58\%), ground glass attenuation areas (50\%) and large nodules (25\%). The halo sign was observed in 9 cases (75\%), cavitations in 3 patients (25\%) and one case presented with the air crescent sign.

Pulmonary lesions in the APA initiates with endobronchial fungal proliferation, followed by vascular invasion and ischemic lung infarct. In this early phase, the histopathological examination demonstrates focus of ischemic lung originating nodules of 1 to $3 \mathrm{~cm}$ with central necrosis and hyphae, and surrounding hemorrhage $[15,17,19]$. This histological finding is demonstrated in the HRCT scan as the halo sign, which was seen in $75 \%$ of our cases.

Cavitations are usually a late finding in patients with APA, and represent the resolution phase of the infection $[5,15,20]$. These findings are seen due to the presence of granulocytes, generally 3-4 days after leukocyte count achieve more than $1,000 / \mathrm{mm}^{3}$. Gefter et al. [8] studying the image findings of 25 patients with APA, identified cavitations in $48 \%$ of the cases, which associated to the air crescent sign in $83 \%$ of patients. All the cases improved after the cavitation was seen. We found cavitations in three patients (25\%), in one of them 34 days after BMT, with neutrophil count of $2,000 / \mathrm{mm}^{3}$, demonstrating signs of bone marrow recovery. In other patient cavitation was found associated to granulocytopenia $\left(70 / \mathrm{mm}^{3}\right)$, 29 days post-BMT.

In conclusion, patients submitted to BMT who present respiratory symptoms and nodules or consolidations with halo sign at the HRCT need to have the diagnosis of invasive pulmonary aspergillosis included in all the post-BMT phases.

\section{References}

1. Krowka M.J., Rosenow E.C. 3rd, Hoagland HC. Pulmonary complications of bone marrow transplantation.Chest 1985;87:237-46.

2. Ettinger N.A., Trulock E.P. Related Articles, Pulmonary considerations of organ transplantation. Part 2. Am Rev Respir Dis 1991;144:213-23.

3. Worthy S.A., Flint J.D., Müller N.L. Pulmonary complications after bone marrow transplantation: high- resolution CT and pathologic findings Radiographics 1997;17:1359-71.

4. De Bock R. Epidemiology of invasive fungal infections in bone marrow transplantation. EORTC Invasive Fungal Infections Cooperative Group. Bone Marrow Transplant 1994;14:1-2.

5. Winer-Muran H.T., Gurney J.W., Bozeman P.M., Krance R.A. Pulmonary complications after bone marrow transplantation. Radiol Clin North Am 1996;34:97-118.

6. Cordonnier C., Bernaudin J.F., Bierling P., et al. Pulmonary complications occurring after allogeneic bone marrow transplantation. A study of 130 consecutive transplanted patients. Cancer 1986;58:1047-54.

7. Feller-Kopman D., Ernst A. The role of bronchoalveolar lavage in the imunocompromised host. Semin Respir Infect 2003; 18:87-94

8. Gefter W.B., Albelda S.M., Talbot G.H., et al. pulmonary aspergillosis and acute leukemia. Limitations in the diagnostic utility of the air crescent sign. Radiology 1985;157:605-10.

9. Graham N.J., Müller N.L., Miller R.R., Shepherd J.D. Intrathoracic complications following allogeneic bone marrow transplantation: CT findings. Radiology 1991;181:153-6.

10. Kim K., Lee M.H., Kim J., et al. Importance of open lung biopsy in the diagnosis of invasive pulmonary aspergillosis in patients with hematologic malignancies. Am J Hematol 2002;71:75-9.

11. Heussel C.P., Kauczor H.U., Heussel G.E., et al. Pneumonia in febrile neutropenic patients and in bone marrow and blood stemcell transplant recipients: use of high-resolution computed tomography. J Clin Oncol 1999;17:796-805.

12. Leung A.N., Gosselin M.V., Napper C.H., et al. Pulmonary infections after bone marrow transplantation: clinical and radiographic findings. Radiology 1999;210:699-710.

13. Jantunen E., Piilonen A., Parkkali T., et al. Diagnostic aspects of invasive Aspergillus infections in allogenic BMT recipients. Bone Marrow Transplantation 2000;25:867-71.

14. Janzen D.L., Adler B.D., Padley S.P., Muller N.L. Diagnostic success of bronchoscopic biopsy in immunocompromised patients with acute pulmonary disease: predictive value of disease distribution as shown on CT. Am J Roentgenol 1993;160:21-4.

15. Mori M., Galvin J.R., Barloon T.J., et al. Fungal pulmonary infections after bone marrow transplantation: evaluation with radiography and CT. Radiology 1991;178:721-6.

16. Oh Y.W., Effmann E.L., Godwin J.D. Pulmonary infections in immunocompromised hosts: the importance of correlating the conventional radiologic appearance with the clinical setting. Radiology 2000;217:647-56.

17. Primack S.L., Muller N.L. High-resolution computed tomography in acute diffuse lung disease in the immunocompromised patient. Radiol Clin North Am 1994;32:731-44.

18. Franquet T., Muller N.L., Gimenez A., et al. Spectrum of Pulmonary Aspergillosis: Histologic, Clinical, and Radiologic Findings. Radio Graphics 2001;21:825-37.

19. Marchiori E., Valiante P.M., Souza Jr. A.S. Nódulos com sinal do halo na aspergilose pulmonar angioinvasiva: correlação da tomografia computadorizada de alta resolução com a anatomopatologia. Radiol Bras 2002;35:195-8.

20. Won H.J., Lee K.S., Cheon J.E., et al. Invasive pulmonary aspergillosis: prediction at thin-section CT in patients with neutropenia-a prospective study. Radiology 1998;208:77782. 